\title{
Short granular chain under vibration: Spontaneous switching of states
}

\author{
Y.-C. Sun (孫宇岑), ${ }^{1,2}$ H.-T. Fei (費祥霆), ${ }^{1}$ P.-C. Huang (黃柏嘉), ${ }^{1,3}$ W.-T. Juan (阮文滔), ${ }^{1,4}$ J.-R. Huang (黃仲仁), ${ }^{2, *}$ \\ and J.-C. Tsai (蔡日強) $)^{1, \dagger}$ \\ ${ }^{1}$ Institute of Physics, Academia Sinica, Taipei 11529, Taiwan \\ ${ }^{2}$ Department of Physics, National Taiwan Normal University, Taipei 10677, Taiwan \\ ${ }^{3}$ Department of Physics, National Taiwan University, Taipei 10617, Taiwan \\ ${ }^{4}$ Integrative Stem Cell Center, China Medical University Hospital, China Medical University, Taichung 40402, Taiwan
}

(Received 25 June 2015; revised manuscript received 31 December 2015; published 2 March 2016)

\begin{abstract}
We study experimentally a short chain of $N(\leqslant 8)$ loosely connected spheres bouncing against a horizontal surface that vibrates sinusoidally at intensity $\Gamma$. Distinct states are identified: a base state of uniform bouncing in-sync with the substrate prevails at low values of $\Gamma$, whereas increasing $\Gamma$ can induce transitions to two excited states with appreciable storage of energy around one or both ends of the chain. We find that, in a transitional window of $\Gamma$, the chain can even switch spontaneously among states, resolving the mystery why different modes of motion can be initiated at the same position in our previous work along a gradient of vibration [Phys. Rev. Lett. 112, 058001 (2014)]. Preliminary interpretations on the parametric dependences and the optimal frequency window for seeing these transitions are offered, based on the microscopic and statistical evidence in our experiments up to date.
\end{abstract}

DOI: 10.1103/PhysRevE.93.032902

\section{INTRODUCTION}

In nature, objects with internal degrees of freedom can exhibit different states in response to external excitations. Examples range from a functional biological cell to just a simple liquid drop on a substrate. In the studies of granular objects, their response to mechanical vibrations has brought a long tradition of scholarly works. Many experiments have focused on the collective behaviors, such as pattern formations [1], segregation and convections [2], diffusivity [3], and Leidenfrost effects and shock waves [4,5], just to name a few. A few previous studies have addressed the different states of vibration with anisotropic rigid objects. The work by Sano and coworkers has reported the occurrence of directional motion with a specially shaped mass at the values of the dimensionless acceleration $\Gamma$ around 1.8 [6]. The simulations and experiments by Kudrolli and Tsimring's groups reveal that a dumbbell dropped onto a substrate vibrating, at $\Gamma$ slightly lower than 1 , can react with different bouncing modes including one with a ratcheting motion [7]. A recent paper by Kubo et al. combines experiments, simulations, and analytical works on the bifurcations of bouncing modes that are coupled with the chirality of the object [8]. The discussions in those studies focus primarily on the change of the translational motion, without influence from internal degrees of freedom. Meanwhile the behaviors of a long chain of beads have been studied for the evolution of knots on a vibrating stage [9] and in many cases used as a metaphor of polymers [10], given its large number of internal freedoms.

\footnotetext{
*jrhuang@ntnu.edu.tw

$\dagger$ jctsai@phys.sinica.edu.tw
}

Published by the American Physical Society under the terms of the Creative Commons Attribution 3.0 License. Further distribution of this work must maintain attribution to the author(s) and the published article's title, journal citation, and DOI.
We have demonstrated in our recent work [11] that a short granular chain, with just a limited degree of internal freedom, can be used as a representation of a soft dissipative object and leads to interesting discoveries along a spatial gradient of vibration: a dramatic divide in its translational motion is found at locations of $\Gamma$ somewhat lower than 1.8. We interpreted the divide as a result of the interaction between the finite size of the object and the spatial gradient. However, the dynamics behind how the chain chooses among multiple states, apparently in a stochastic fashion, remains largely unexplained. In our current work, with the spatial gradient removed, we characterize the spontaneous switching of the states and the transitions, from both microscopic and statistical points of view.

\section{SETUP AND IMAGING}

As shown in Fig. 1, we impose uniform sinusoidal vibrations to a chain of $N$ loosely connected metal spheres [Fig. 1(a)]. The main control parameter is $\Gamma$, the peak acceleration of the base normalized by the gravitational acceleration $g$, and the frequency $f \sim 25 \mathrm{~Hz}$ typically. Our mechanical shakers are built by a local manufacturer (Vibration Source Technology, Co., Taipei, Taiwan) and are feedback controlled. The chain is partially confined by either two acrylic plates in parallel with a separation slightly larger than the particle diameter $d$, as shown in the linear setup in Fig. 1(b), or by concentric cylinders with the same gap width, as the circular setup in Fig. 1(c). The linear setup has two versions: L1 is $30 \mathrm{~cm}$-long with a straight bottom, while the miniature L2 has a slightly concave bottom with a radius of curvature $20 \mathrm{~cm}$ so that the chain never goes beyond the arch of $8 \mathrm{~cm}$. The circular version is designed specially for statistical experiments, so that observations will not be interrupted as it would occur when the chain reaches either end of a linear channel. Effects of the curvature are negligible, for that the expected bending to comply with the channel is only at the order of $10^{-2}$ radian between adjacent particles. For both the linear and the circular versions, the bottom of the channel is made of acrylic and 
(a)
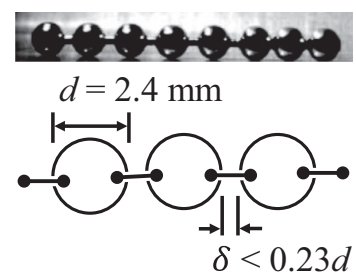

(b)

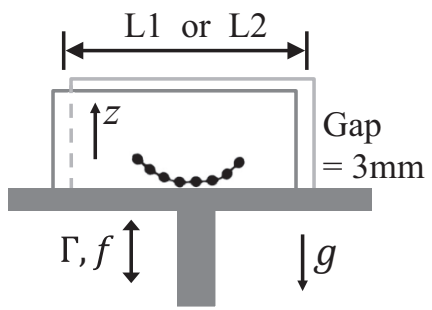

(c)

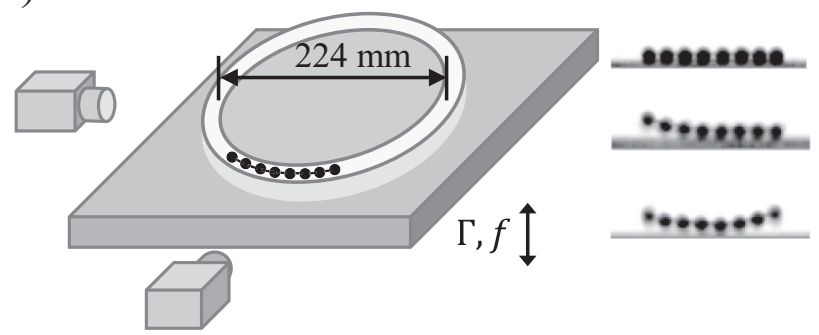

FIG. 1. Schematics of experimental setup (not to scale). (a) Photograph of a typical chain used in the experiment and the relevant dimensions: the sphere diameter $d=2.4 \mathrm{~mm}$ and the linkage distance $\delta<0.23 d$. (b) The linear version, primarily for microscopic observations from the front view. (c) The circular version, with two in-sync cameras oriented in orthogonal directions ensuring continuous long-time recordings. The photographs on the right show examples of the optical average over a vibration cycle, described in main text, for states 0,1 , and 2 in a top-down sequence.

is roughened at the scale of $0.1 \mathrm{~mm}$. We use diffusive back lighting to image the chain in all cases.

We find that the chain in these quasi-two-dimensional (2D) setups faithfully reproduce all key features especially the different states (referred to as modes) in the previous three-dimensional (3D) experiments [11]: at values of $\Gamma$ slightly larger than 1 , the chain remains essentially flat even if it does leave the substrate periodically; in a substantial range of $\Gamma$, the movements appear to be dominated by the swinging of one or two end(s) of the chain; see also our online Supplemental Materials for video demonstrations [12] using our current setup.

To understand the dynamics of the states and their transitions, we take high-speed videos of the chain. By zooming in to the magnification of 100 pixels across the particle, computer-aided centroid algorithm allows us to detect the center of individual particles to the sensitivity at the order of $O(10 \mu \mathrm{m})$ [13]. Simply by visual examination of the high-speed videos, one could also see that the chain takes off from the substrate just once in every vibration cycle and that, in the presence of State 1 or 2, the chain spends a considerable period of time with a significant degree of bending, throughout the range of $\Gamma$ in our scope of discussion. This fact provides a convenient alternative to recognize the state without resorting to high-speed imaging: One could set the imaging exposure time approximately equal to the vibration period and obtain the optically averaged images like those shown on the right-hand side of Fig. 1(c). Our computer code then divides the image of the chain into $N$ segments, calculates the intensity-weighted

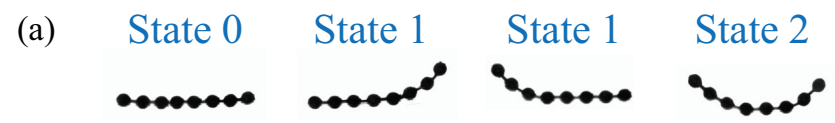

(b) $\left[\mathrm{mm}^{2}\right]$

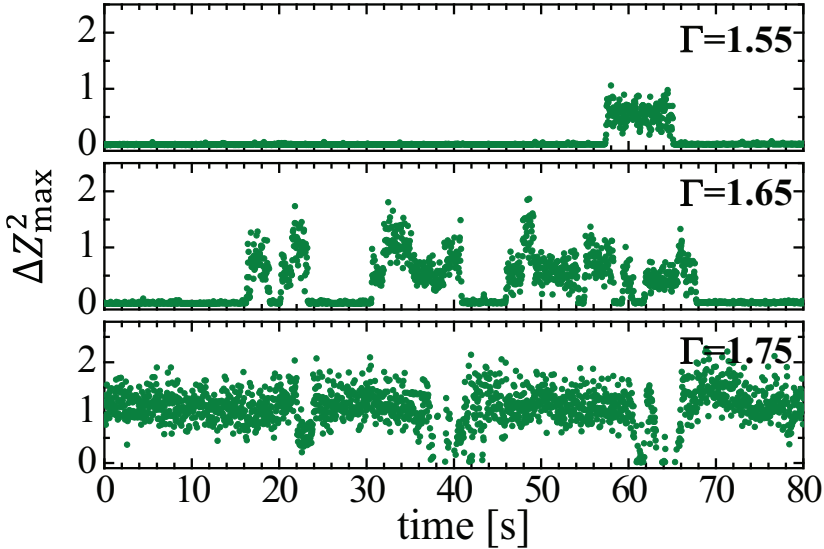

(c) $\left[\mathrm{mm}^{2}\right]$

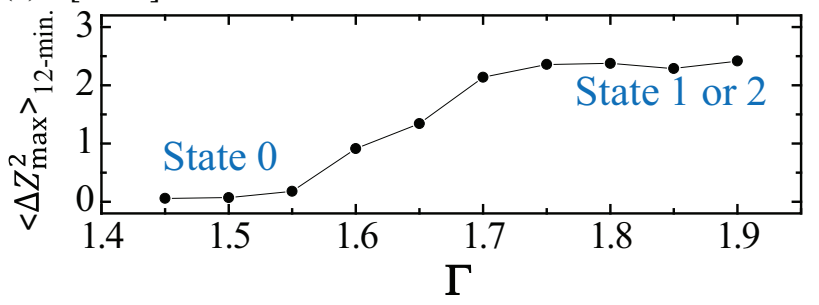

FIG. 2. Illustration of the transition, over time or the control parameter $\Gamma$, with $N=8$ : (a) Snapshot of the three states: state 0 with the chain being essentially flat, state 1 with the one end bending upwards, and state 2 with both ends upwards, captured upon their maximal bending. (b) Time series of $\Delta z_{\max }^{2}$ at $\Gamma=1.6,1.65$, and 1.7 , respectively, at the same frequency $f=25 \mathrm{~Hz}$. (c) The 12-min average of $\Delta z_{\mathrm{max}}^{2}$, at different values of $\Gamma$ with the same frequency.

centers of each, and makes the fully automated judgment on the chain as in one of the three "states" by comparing the heights of the first and last to the one (or two) at the center. One great advantage of this algorithm is that it demands only the normal video frame rates, so that we can make continuous recordings in practically unrestricted lengths on which our statistical analyses are based. Although the judgments inevitably involve a threshold $h_{c}$ in making the comparison against noises, most of the statistics have been verified as reasonably insensitive to the choice of $h_{c}$ (typically $d / 4$, as detailed in Ref. [13]), except for certain limitations towards the small-amplitude limit of our experiments, which will be revisited in Sec. IV B.

\section{MAIN CHARACTERISTICS}

Before describing our particle-tracking and statistical analyses, it is helpful to demonstrate the spontaneous switching with data from high-speed imaging of a chain in our long linear channel (setup L1). Multiple snapshots are obtained within each vibration cycle, and Fig. 2(a) demonstrates the front views of different states at their maximal bending. For each snapshot, we fit each snapshot of the chain with an $N$-degree polynomial, 
find its best fit $z_{N}(x)$, and quantify the bending by

$$
\Delta z^{2} \equiv \int\left[z_{N}(x)-Z_{c}\right]^{2} d x / \int d x
$$

in which $Z_{c} \equiv \int z_{N}(x) d x / \int d x$. As a rough indicator of the "state," here we show in Fig. 2(b) the time series of $\Delta z_{\max }^{2}$, which stands for the peak value of $\Delta z^{2}$ found in multiple snapshots within each vibration cycle. Even though our images need to accommodate the long channel and therefore do not have the pixel resolution required for a precision particle tracking (as in the next subsection), the rise and fall of $\Delta z_{\max }^{2}$ serves as an effective indicator of the chain alternating between State 0 and the other two "excited states." Furthermore, Fig. 2(c) displays the values of $\Delta z_{\max }^{2}$ averaged over 1800 vibration cycles for experiments of different values of $\Gamma$, showing the trend towards a saturation as $\Gamma$ increases. However, this indicator does not differentiate the contribution of State 1 or State 2.

In Sec. III A, by zooming in to the highest pixel resolution available, we examine the motion of these individual particles, quantifying the energy and dissipations in different states, and establishing the time scales of the spontaneous switching, at a fixed set of parameters $N=8, \Gamma=1.65$, and $f=25 \mathrm{~Hz}$. In Sec. III B we characterize by statistical analyses the dependence over $N, \Gamma$, and $f$ for these phenomena, with full distinctions among all three states.

\section{A. Particle dynamics}

In this section, we describe results from high-resolution imaging, with experiments performed with setup L2 to suppress the horizontal displacements with its small curvature. The suppression of horizontal displacements allows the zoomin imaging at our full pixel resolution. These experiments are performed at a fixed set of parameter $N=8, \Gamma=1.65$, and $f=25 \mathrm{~Hz}$.

Figure 3 demonstrates trajectories and speeds of individual particles in a transition from State 0 to State 1, in a typical pace of about ten cycles. Trajectories are presented as potential energies (PEs), speeds as in kinetic energies (KEs, the end particles only), with the sum of energies of all particles $(\mathrm{PE}+\mathrm{KE})$ normalized by the total number of particles $N=8$ for the ease of comparisons. Figure 3 also demonstrates the quality of the particle tracking: the data for the height of individual particles $\left(Z_{i}\right)$ appear continuous, and the velocities $\left(V_{i}\right)$ derived from frame-to-frame subtractions of $Z_{i}$ are reasonably smooth between collisions, as the combined results of the high frame rate $(2500 \mathrm{fps})$ and a sufficiently high pixel resolution on the zoom-in images.

Notably, the energies become significantly uneven as the chain switches from State 0 to State 1. Quantitatively, the energy carried by a particle at the swinging end of State 1 is at the order of $g \times 3 \mathrm{~mm} \approx 0.03(\mathrm{~m} / \mathrm{s})^{2}$ per particle, while the sharp dips on the curve of the total energy suggest that the energy loss upon each landing is around $0.01(\mathrm{~m} / \mathrm{s})^{2}$ per particle. Nevertheless, one shared feature between State 0 and State 1 is that the descent of the total energy is about twice steeper than its rising. This fact suggests a notable asymmetry that can be related to the ways how the chain loses and regains energies, as will be shown in the next figure.

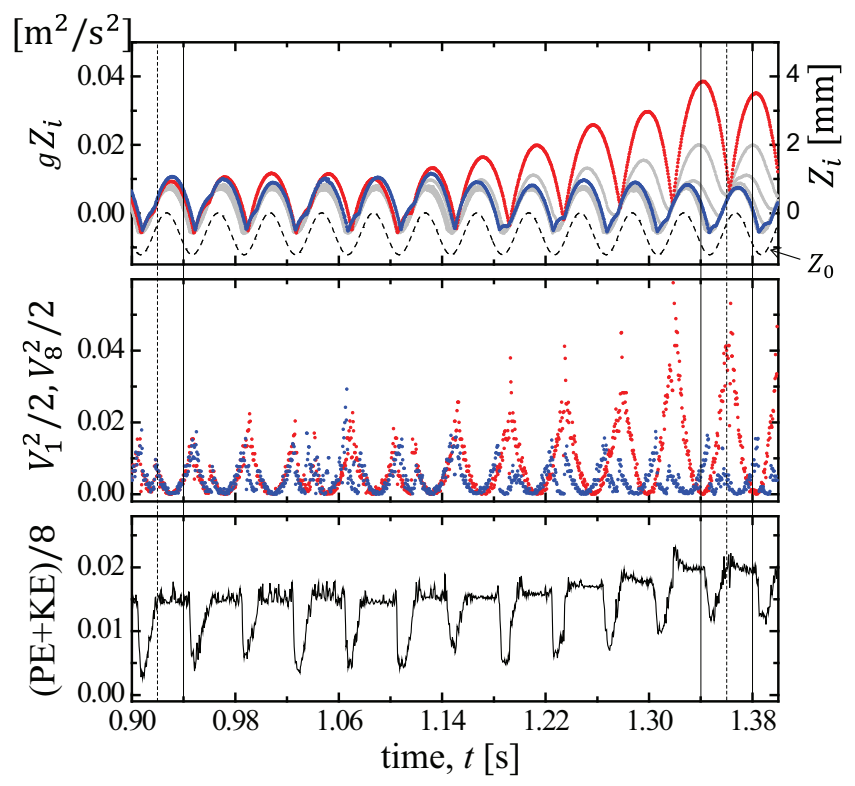

FIG. 3. Time-resolved particle trajectories and energies during a spontaneous transition from State 0 to State 1 , with $\Gamma=1.65$ and $f=25 \mathrm{~Hz}$. The potential energies (PEs) of each individual particles are expressed as $g Z_{i}$ in which the two end particles are represented in red and blue on the top panel, the kinetic energies (KEs) of the two end particles as $V_{1}^{2} / 2$ and $V_{8}^{2} / 2$, and the total energy as $(\mathrm{PE}+\mathrm{KE}) / N$ for assessing the dissipations. The particle mass is omitted in these expressions. Motion of the substrate $Z_{0}$ is indicated on the top panel (dashed curve).

By zooming in the response cycles of each state, Fig. 4 shows the contact dynamics. While Fig. 4(a) further magnifies the particle motion presented in the preceding figure with the substrate movement subtracted, Fig. 4(b) demonstrates the dramatic difference in how the chain interacts with the substrate: In State 0, the trajectories suggest that all particles land on the substrate within a narrow time window (roughly the second eighth of the vibration cycle presented with this graph). The rebounce of the two end particles is clearly identifiable, whereas the inner particles stay close to the substrate for nearly half of the vibration cycle. In contrast, the landings of individual particles for State 1 spread much wider in time: starting around the same time relative to the vibration cycle as in State 0 , these landings occur sequentially for over half of the vibration cycle and end with the ballistic impact of the end particle. We display four snapshots in Fig. 4(c) to demonstrate the time sequence of the head-swinging movement, supplementing our previous interpretations on the rapid horizontal ratcheting associated with State 1 [11].

Even though some details of the contacts might remain unresolved with our current image resolution, cross-referencing Fig. 3(c) and Fig. 4(b) reveals that, in both State 0 and State 1, the chain spends roughly half of the vibration cycle in a free flight: starting at around $t=0.92$ and $1.36 \mathrm{~s}$, for instance, as also shown by the vertical dashed lines where the total energy start to plateau (with some noises reflecting measurement errors, such as neglected energies in rotation or horizontal movements). In addition, these zoom-in observations support 


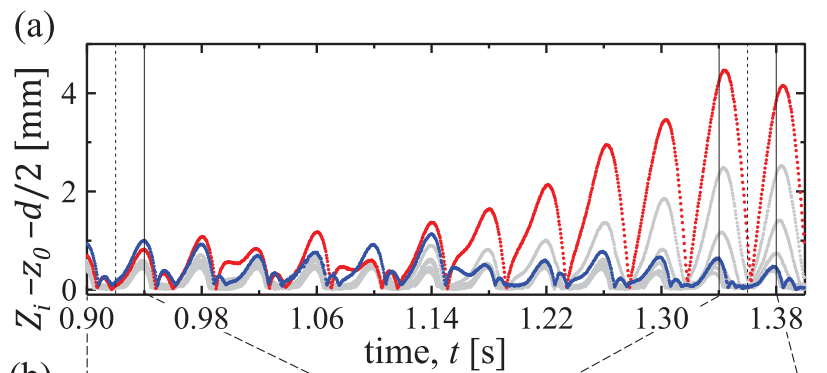

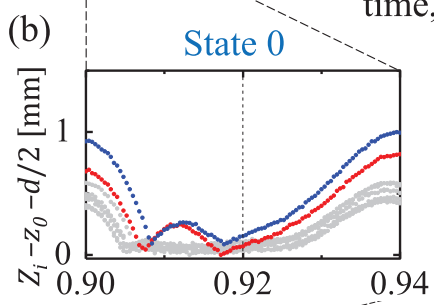

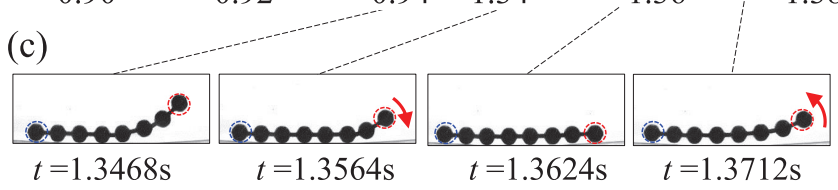

FIG. 4. Close-up observations of the two states, with substrate movement subtracted, from the data presented in Fig. 3. (a) The subtracted trajectories, indicating the distance of individual particles to the substrate. (b) Magnified display of one vibration cycle in State 0 and State 1, respectively. (c) High-speed snapshots of the chain at State 1, with two end particles marked by red and blue dashed circles, analogous to (a) and (b). The arrows indicate the direction of the end particle.

our preceding interpretation on the steep descent in the total energy: The dissipations occurs mostly with the first landings within about one-eighth of the vibration cycle. The slower rise of the total energy in Fig. 3(c) reflects the substrate's supply of energy to "grounded" particles by pushing them upwards in a relatively more gradual manner, lasting about one-fourth of the vibration cycle, until the free flights take place; see Fig. 3(a) for the phase of substrate movements as well. Further thoughts on the relation of these phenomena to the takeoff and landing of a simple point mass will be discussed in Sec. IV A and with numerics supplied in Appendix.

We show in Fig. 5 the spontaneous switching among all three states, at the same experimental condition as in Fig. 3 for a much longer timescale. The energies here are shown by the average over each vibration cycle. The alternation of averaged height of the end particles, or equivalently their PE, stands outs as the most effective indicator of the switching among three states.

One might expect the total energy to exhibit three distinct levels, instead of just two as shown by the bottom graph. This apparent degeneracy in energy can be explained as we take into account a bias in State 1 due to the use of a slightly curved channel (setup L2): As reported in Ref. [11], the chain exhibits a strong tendency to move towards the active side at State 1 . While the slight curvature successfully stops the chain from moving out of our imaging zone, one of the unintended consequences is that the PE baseline for a particle at the swinging end in State 1 shifts upward as the chain moves uphill (as shown by the video images), at a typical amount

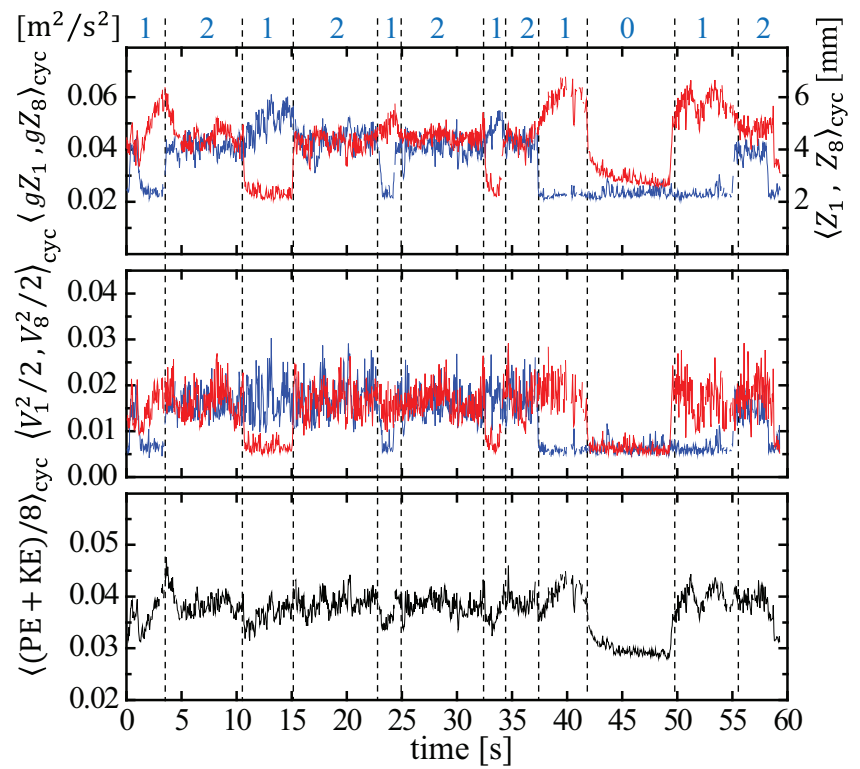

FIG. 5. Spontaneous switching among the three states, represented by the relevant mean energies, in analogy to Fig. 3 but for a much larger time scale: the PE and KE of the two end particles, and the sum of energies along the entire chain, with a bracket $\langle\cdots\rangle_{\text {cyc }}$ standing for computing the mean over exactly one vibration cycle. The numbers 0,1 , and 2 on the top indicate the state of the chain, switching with intervals marked by the dashed lines.

of $g \times 2 \mathrm{~mm} \approx 0.02(\mathrm{~m} / \mathrm{s})^{2}$ per particle per unit mass, as is also shown by the gradual rise of the height of one of the end particle (but not the other one that stays around the valley of the channel) during State 1. The rise roughly compensates for the expected energy gap between State 1 and State 2, which might have appeared as a a few sharp steps in PE and KE upon the activation of State 1 but are evened out later as the chain climb upwards. Some other features in the curve of total energy can also be consistently explained by this effect, such as the slow descent $(t \approx 42 \mathrm{~s})$ versus the steep rise $(t \approx 50 \mathrm{~s})$, because the uphill movement of the chain at State 1 is inherently fast in comparison to the gradual sliding towards the valley of the channel when this head-swinging ceases to exists (in State 0 or becomes symmetric as in State 2).

Most importantly, the information from Figs. 5 and 3 combined shows that the height alternations are unambiguous and that the switching of states, typically within 10 vibration cycles, is abrupt in comparison to the durations of each state, which could last a few seconds or even much longer [12]. These are empirical facts that justify the automated state recognition, described in Sec. II, based on which we are able to characterize the transitions by statistical analyses, without knowing the details of particle motion.

\section{B. Statistical descriptions}

The use of the circular channel, combined with the automated criterion of state described in Sec. II, permits long-time statistical studies of the states and the transition over various factors. We use the fraction of counting among different states during a long recording (typically $10^{5}$ to $10^{6}$ vibration cycles) to assess the likelihood of a chain being in 


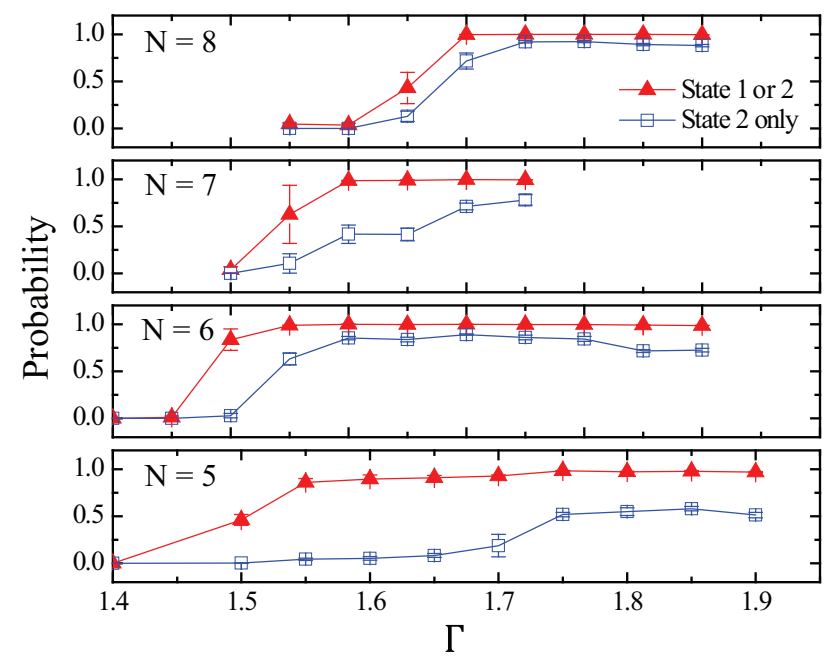

FIG. 6. Comparison of chains at different lengths with $N=$ $5,6,7$, and 8 , based on the probabilities of finding a chain at the specified state(s) during a recording of 65000 consecutive vibration cycles $(=2600 \mathrm{~s})$ at $f=25 \mathrm{~Hz}$, as functions of $\Gamma$.

specific states, as a way to characterize the transition over our control parameters ( $\Gamma$ and $f$ ) and to compare the behaviors of a chain at different lengths.

\section{Dependence on chain length}

Figure 6 compares the transitions over $\Gamma$, for different chain lengths. One may refer to the rise of the likelihood for State 1 and 2 combined as a first sign, and that for State 2 as the second that completes the transition.

The first notable feature is a trend that the first sign seems to occur at lower values of $\Gamma$ for a shorter chain. We will discuss its relation with dissipation in Sec. IV A. We also note that, for $N=5$, the separation between the two curves is enlarged dramatically; we believe that this suggests a length scale below which the two ends of the chain are closely coupled: activation of one side of the five-particle chain somehow hinders the energy accumulation at the other, so that State 2 becomes hard to maintain, in comparison to the cases for longer chains.

\section{Dependence on frequency}

Figure 7(a) shows that the transitions over $\Gamma$ at three frequencies 15,25 , and $35 \mathrm{~Hz}$ are qualitatively similar but do exhibit certain changes over frequency. Complications arise as we vary the frequency further away from our typical values: At low frequencies, the substrate vibration can become larger that the particle diameter $d$ and the chain even flips over sometimes, creating difficulties in defining the states. At high frequencies, the amplitudes of the chain bending are small so that the automated criterion of states becomes sensitive to the threshold value $h_{c}$. As a result, our observations seem to reflect an optimal range of frequency, as shown in Fig. 7(b), for seeing the state transitions under these practical constraints. In Sec. IV B, we will revisit the physical meanings behind such a frequency window, in terms of the energy scales involved.
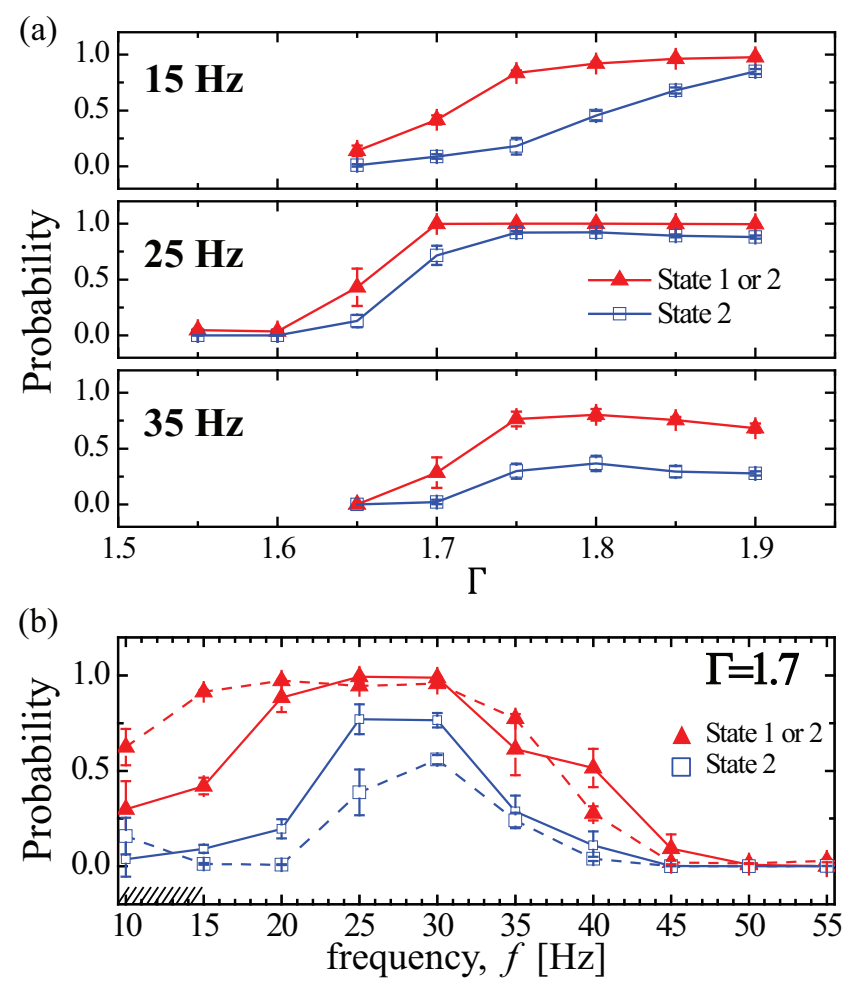

FIG. 7. Probabilities of finding a chain at the specified state(s) during long recordings: dependence on the two control parameters: (a) Dependence on $\Gamma$ at $f=15,25$, and $35 \mathrm{~Hz}$, respectively, for a chain with $N=8$. (b) Dependence on frequency $f$ at $\Gamma=1.7$ for the same chain. The additional data using a chain with $N=5$ are also included with dashed lines. Below $15 \mathrm{~Hz}$, we use large error bars to convey the message that the excessive vibration amplitudes makes the states sometimes ill-defined, as explained in main text. The length of recording times varies in these data but is in general at the order of $O(10000)$ vibration cycles.

\section{DISCUSSION}

In this section, we discuss the effects of various factors on the observed transitions, based on the microscopic and statistical evidence established so far.

\section{A. Transitions over $\Gamma$, with internal dissipations}

Empirical facts shown in Sec. III B reveal that $\Gamma$ is indeed the leading factor for the transition from the dominance of State 0 to that of the excited states, and that the sharpest changes occur the values of $\Gamma$ from around 1.5 to 1.7 . It is helpful to consider the behavior of a hypothetical point mass taking off from a substrate vibrating sinusoidally. Using results from elementary calculations which we adapt partially in the Appendix, we note that the phase of the landing, as a function of $\Gamma$ only, is quite close to the valley of the driving motion at $\Gamma \approx 1.7$, above which the landing occurs in the rising phase of vibration. Therefore, given the empirical fact that State 0 typically loses all energies before the next cycle begins, State 0 can be expected to be quite stable at values of $\Gamma$ well below 1.7. Above this value, chattering of the end particles (which are likely to be more excited than the inner ones) can extend to the rising phase and has a chance to be amplified 
to a large-scale swinging motion; we have made available online our illustrations with $N=8$ as $\Gamma$ approaches 1.65 [12]. However, the rising of an end particle requires overcoming the restraint from the rest of the chain, therefore its success appears stochastic: For instance, Fig. 6 shows that, at $\Gamma=1.65$, the success rate for a departure from State 0 to excited states is somewhat below $50 \%$.

In addition, evidence shows that dissipations due to the interparticles interaction are quite appreciable: even though our estimate suggests that our particles interact with the substrate with a restitution coefficient around 0.5 , we have found that the mechanical connections between particles dissipate much more energies than just the substrate collisions do; see the Appendix for further discussion. We believe that this is the main reason why a theory of a fully dissipative point mass seems to establish a baseline for understanding the characteristic $\Gamma$ for seeing the first sign of transition. By the same token, it is also reasonable that, with the chain length $N$ reduced, the chain is generally less dissipative so that more energy survives the impact; that seems to explain why the curve of the first transition shifts towards lower values of $\Gamma$ with a shorter chain in Fig. 6: larger amplitudes of chattering are more likely to be amplified to become the precursor of the state transition.

The limited degree of freedoms of a short chain offers the possibilities that the problem may be treated with existing tools in the studies of dynamical systems. For instance, what triggers the instability leading to the accumulation of energy towards the end(s) of the chain? Over the change of $\Gamma$, is it possible to interpret the crossover from the dominance of State 0 to that of the two head-swinging states as the shrinkage of an existing locking region in the phase space [14], along with the creation and growth of new ones? This may be one of the issues worthy of attention.

\section{B. Role of frequency and amplitude}

Even though Fig. 7 shows that the change of behavior is not as sensitive to frequency as to the dimensionless acceleration $\Gamma$, one should anticipate that the scenarios might change qualitatively, if we make a substantial change on frequency $f$, as we assess below.

It is helpful to define a characteristic kinetic energy per unit mass as

$$
\epsilon_{k}=\left(\omega Z^{(0)}\right)^{2}=(\Gamma g / \omega)^{2},
$$

where $\omega=2 \pi f$ and $Z^{(0)}$ stands for the amplitude of a sinusoidally vibrating substrate. This energy is, to certain degrees, analogous to a granular temperature imposed by the vibration. Dimensional analyses suggest that the height fluctuations among the these loosely connected particles should be around $\delta z=\epsilon_{k} / g \approx 1 \mathrm{~mm}$ at $\Gamma=1.65$ and $f=$ $25 \mathrm{~Hz}$, in consistence with our observations of the trajectories displayed in Fig. 3. However, as $\epsilon_{k}$ goes inversely with $\omega^{2}$, one could anticipate that the amplitude of the chain bending decreases accordingly and becomes hard to detect: The truth is that, at $35 \mathrm{~Hz}$, the chain bending is so small that outcomes of the automated judgment of states become sensitive to the threshold value $h_{c}$ we adopt. This contributes partially to the underestimation of the true population of events for State 1 and 2 in Fig. 7(a), although the prevalence of these two states with increasing $\Gamma$ is still indisputable. In addition to the issue of detectability, we should point out that, as energy level $\epsilon_{k}$ scales down with increasing frequencies, the influence of local asperities and dirts become substantial and can render the attempts to extend our experiment towards its high-frequency limit somewhat impractical.

The low-frequency limit presents a different issue: As the particles are connected by constraints, one should expect an upper limit in the fluctuations of potential energies associated with the height differences among particles

$$
\epsilon^{\max }=g \times \delta z^{\max }
$$

per particle per unit mass, with $\delta z^{\max } \sim O(1) d$ set by the constraint between adjacent particles. One could anticipate that, as $\epsilon_{k}$ scales up with decreasing frequency, this kinetic energy eventually exceeds what can be accommodated by the bending of the chain [15]. In fact, as we point out in Sec. III B, the chain does flip over at low frequencies and presents behaviors beyond what are intended to be described by the three states. Therefore, the optimal range of frequency for seeing the state-switching behaviors reflects a matching of the kinetic energy $\epsilon_{k}$ as close to $\epsilon^{\max }$ as possible, such that the appearance of States 1 and 2 can be identified unambiguously with ease.

\section{SUMMARY}

In this work, we study systematically the behaviors of a short granular chain in response to a sinusoidal vibration of uniform but tunable intensity. The quasi-2D setup reproduces the multiple states and transitions that we previously discovered on open channels in three dimensions. In certain ranges of excitation intensity, the chain exhibits spontaneous switching among different states without the excitation ever changed. This coexistence of possibilities in different states explains why, in our previous work with a spatial gradient of excitation [11], qualitatively different bouncing modes can occur stochastically at the same initial position and, in turn, create the dramatic spatial divide. Microscopic behaviors are characterized by high-speed imaging and precision particle tracking, helping us understand the energies and dissipations in detail. Statistical analyses based on the long series of images at low frame rates, on the other hand, provide the overviews on how the chain changes its behaviors over the two control parameters: the dimensionless acceleration $\Gamma$ and the frequency $f$.

Unlike a simple bouncing ball for which $\Gamma$ is the only control parameter (plus a coefficient for rebounce), the linkages along the chain creates substantial dissipations that seem to create certain stabilities for the different states despite their switching, making the rebounce less relevant, and brings in a dependence on frequency $f$ as well. By dimensional analyses, we interpret the apparent frequency window for the observation of state switching as the matching of energy scale between the kinetic energies imposed by the substrate vibrations and the fluctuations of gravitational energy along the chain.

In our subsequent reports, we will offer an alternative interpretation of the frequency window as the matching 
between the vibration period to a characteristic dissipation time determined from the inter-article connections, based on a model with a series of linearly coupled point masses. Our subsequent works also characterize the distinct modes of horizontal displacement that not only reflect the different states but also switch spontaneously. We believe that deeper looks into these switching states and their displacements help elucidate how the internal freedom of a soft object, in general, reacts to external driving and in turn produces macroscopic consequences.

\section{ACKNOWLEDGMENTS}

The authors acknowledge fruitful exchanges with C.-K. Chan, M.-R. Chou, C.-Y. Tao, and our former collaborator W.-T. Lin, assistance from the AS-IoP Machine Shop, and grant support from Institute of Physics, Academia Sinica (ASIoP) and Ministry of Science and Technology (under NSC1002112-M-003-001-MY3 and MOST104-2112-M-001-031) in Taipei, Taiwan.

\section{APPENDIX}

We assess the particle bouncing from both theoretical and experimental point of views, with some of the results shown in Fig. 8. In panels (a) and (b), we show the predictions for the flight trajectory, $z(t)$, of a noncohesive point mass resting and taking off from a vibrating plane at $Z^{(0)} \sin (2 \pi f t)$, using elementary calculations; the phase of landing $\left(\phi_{L}\right)$ is numerically determined by solving the intersection of the parabolic flight with the sinusoidal vibration $[13,16]$. Note that the phase $\phi=2 \pi f t$ defines a dimensionless time and that these results are frequency-independent. They describe the behavior of one particle at the fully damped limit (with no rebounce).

On the other hand, our observations reveal that particles along the chain do rebounce to a certain degree: The measured trajectory for an end particle in State 0, as shown in panel (c), is presented in an inertial coordinate defined by

$$
Z_{1}^{\prime}[t]=Z_{1}[t]-\left[Z_{01}+V_{01}\left(t-t_{1}\right)\right]
$$

in which $Z_{01}$ and $V_{01}$ represent the position and velocity of the substrate upon the impact at time $t_{1}$. The best fits with a parabola of height $h_{0}$ and $h_{1}$ are found, respectively, before and after the time of impact $t_{1}$. We use $e=\left(h_{1} / h_{0}\right)^{0.5}$ as our estimate of restitution coefficient, giving $e \approx 0.55 \pm 0.07$,
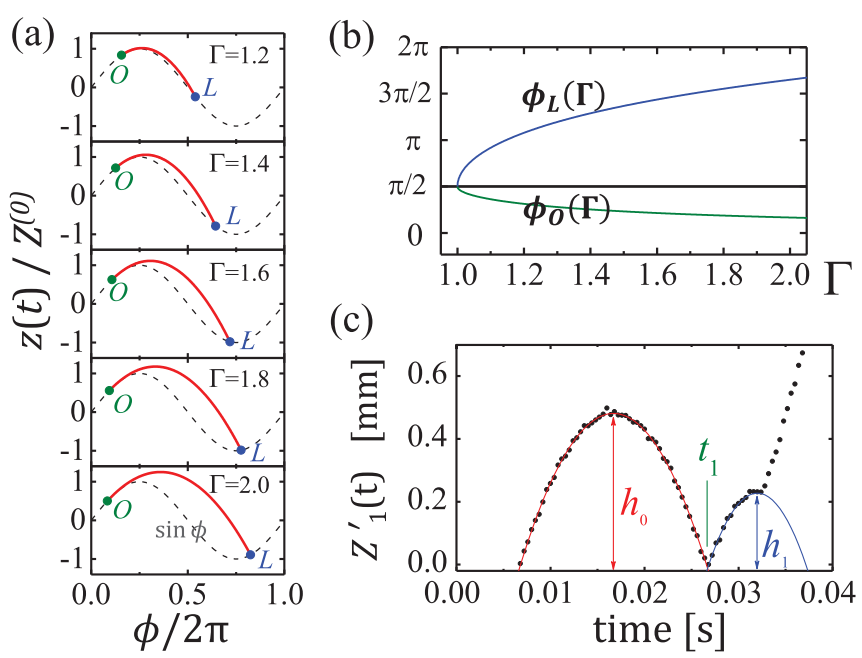

(c)

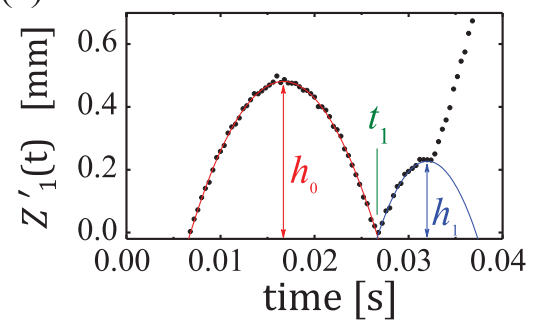

FIG. 8. Numerics and empirical assessments on the particle bouncing against a sinusoidally vibrating substrate. (a) Sample trajectories of $z(t)$ for a noncohesive point mass taking off from (O) and landing on (L) a vibrating substrate of different $\Gamma$, in a normalized coordinate. (b) The functions $\phi_{O}(\Gamma)=\arcsin (1 / \Gamma)$ that describe the takeoff phase and the numerically determined $\phi_{L}(\Gamma)$ for the phase at landing. (c) Empirically measured trajectory of an end particle in an eight-particle chain, above a substrate vibrating at $\Gamma=1.45$ and $f=25 \mathrm{~Hz}$. The data (black dots) are presented and fitted in an inertial frame described in the Appendix.

which is consistent with our other data obtained independently from dropping a single ball cut from the chain (not shown).

However, we also find that particles other than the end spheres loose much more energy, presumably because of being constrained by two linkages rather than just one: For the fourth or fifth sphere in an eight-particle chain, only $20 \%$ of the landings can be identified with a recognizable rebounce (with amplitudes never exceeding those of the end particles), suggesting that over $95 \%$ of their kinetic energies are lost immediately upon the landing. We believe that this is why the simple model of parabolic flight, with the rebounce totally ignored, still gives a reasonable baseline for assessing the behavior of a chain at different values of $\Gamma$ before it departs from State 0 .
[1] P. B. Umbanhowar, F. Melo, and H. L. Swinney, Nature (London) 382, 793 (1996).

[2] J. B. Knight, H. M. Jaeger, and S. R. Nagel, Phys. Rev. Lett. 70, 3728 (1993); J. M. Ottino and D. V. Khakhar, Annu. Rev. Fluid Mech. 32, 55 (2000); P. Eshuis, D. van der Meer, M. Alam, H. J. van Gerner, K. van der Weele, and D. Lohse, Phys. Rev. Lett. 104, 038001 (2010).

[3] P. M. Reis, R. A. Ingale, and M. D. Shattuck, Phys. Rev. E 75, 051311 (2007); R. D. Wildman, J. Beecham, and T. L. Freeman, Eur. Phys. J. Special Topics 179, 5 (2010); V. Yadav and A. Kudrolli, Eur. Phys. J. E 35, 104 (2012).
[4] P. Eshuis , K. van der Weele, D. van der Meer, and D. Lohse, Phys. Rev. Lett. 95, 258001 (2005).

[5] J. A. Perez, S. B. Kachuck, and G. A. Voth, Phys. Rev. E 78, 041309 (2008).

[6] D. Yamada, T. Hondou, and M. Sano, Phys. Rev. E 67, 040301 (2003).

[7] D. Volfson, A. Kudrolli, and L. S. Tsimring, Phys. Rev. E 70, 051312 (2004); S. Dorbolo, D. Volfson, L. Tsimring, and A. Kudrolli, Phys. Rev. Lett. 95, 044101 (2005).

[8] Y. Kubo, S. Inagaki, M. Ichikawa, and K. Yoshikawa, Phys. Rev. E 91, 052905 (2015). 
[9] E. Ben-Naim, Z. A. Daya, P. Vorobieff, and R. E. Ecke, Phys. Rev. Lett. 86, 1414 (2001).

[10] K. Safford, Y. Kantor, M. Kardar, and A. Kudrolli, Phys. Rev. E 79, 061304 (2009).

[11] W.-T. Lin, Y.-C. Sun, C.-C. Chang, Y.-C. Lin, C.-W. Peng, W.-T. Juan, and J.-C. Tsai, Phys. Rev. Lett. 112, 058001 (2014).

[12] http://www.phys.sinica.edu.tw/jctsai/switching/.

[13] Y.-C. Sun, Master's thesis, National Taiwan Normal University, Taipei, June 2015. Also available through the website in Ref. [12].
[14] J. M. Luck and A. Mehta, Phys. Rev. E 48, 3988 (1993); see also relevant backgrounds in N. B. Tufillaro, T. M. Mello, Y. M. Choi, and A. M. Albano, J. Phys. France 47, 1477 (1986); A. Mehta and J. M. Luck, Phys. Rev. Lett. 65, 393 (1990).

[15] Comparing the two energy scales is in the same spirit of considering the shaking parameter $S$ in Ref. [4], in which $S=\epsilon_{k} / \epsilon_{c}$ and the author defines $\epsilon_{c}$ as $g d$.

[16] J. J. Barroso, M. V. Carneiro, and E. E. N. Macau, Phys. Rev. E 79, 026206 (2009). 